\title{
MicroRNA-26a-5p: multiple functions, multiple possibilities - a mini-review
}

\author{
Paweł Zapolnik ${ }^{1, A-D \oplus}$, Beata Zapolnik ${ }^{2, E-F} \oplus$ \\ ${ }^{1}$ Students' Scientific Association of Clinical Genetics, Department of Clinical Genetics, Medical College, University of \\ Rzeszów, Poland \\ ${ }^{2}$ Department of Allergology and Cystic Fibrosis, Clinical Regional Hospital No. 2, Rzeszów, Poland \\ $A$ - Research concept and design, B - Collection and/or assembly of data, C - Data analysis and interpretation, \\ $D$ - Writing the article, E - Critical revision of the article, $F$ - Final approval of article
}

Zapolnik P, Zapolnik B. MicroRNA-26a-5p: multiple functions, multiple possibilities- a mini-review. J Pre-Clin Clin Res. 2020; 14(4): 130-133. doi: $10.26444 / j p c c r / 128009$

\begin{abstract}
Introduction. MicroRNA are small RNA molecules about 22 nucleotides long that do not encode proteins. Their function is to change the expression of proteins by attaching to their mRNA. They participate in various biological processes and pathogenesis of many diseases, including cancer. MicroRNA-26a-5p (miR-26a-5p) takes part in developing many disease entities and may become a potential therapeutic agent.

Objective. The article aims to present the current knowledge on miR-26a-5p and consider the possible therapeutic use of this molecule.

State of knowledge. Many studies on cell lines and model organisms indicate the suppressive function of miR-26a-5p in such neoplasms as breast cancer, pancreatic cancer, prostate cancer, thyroid cancer, or acute myeloid leukemia. In turn, in osteosarcoma cells, miR-26a-5p has an oncogenic character. miR-26a-5p is also associated with other diseases. Many authors have shown that this molecule is related to such entities as rheumatoid arthritis, osteoarthritis, preeclampsia, myocardial injury, or diabetic nephropathy.

Conclusions. Understanding the functions of miR-26a-5p and its participation in human diseases' pathogenesis gives the possibility of the therapeutic use of this molecule in the future. However, knowledge of this subject is still limited. Further research to assess the efficacy and safety of the therapeutic use of miR-26a-5p is mandatory.
\end{abstract}

\section{Key words}

review, neoplasms, microRNAs, molecular targeted therapy

\section{INTRODUCTION}

MicroRNA (miRNA) are small, evolutionarily conserved, non-coding RNA molecules, approximately 22 nucleotides in length, which participate in the post-transcriptional alteration of protein expression by attaching to corresponding mRNA molecules. Due to this, they take part in various cellular processes, including proliferation and apoptosis $[1,2,3,4]$. Dysregulation of the relevant miRNAs may be associated with cell proliferation and oncogenesis. Recent studies indicate that there are numerous miRNAs with a tumour suppressor or oncogenic function [5]. At present, MicroRNA-26a-5p (miR-26a-5p) is a little-known molecule although its participation in both neoplastic and nonneoplastic processes is understood. There are reports of a significant suppressive role of miR-26a-5p in various types of cancer [6]. Due to its function of regulating the expression of proteins in a post-transcriptional manner, miR-26a-5p can be used therapeutically in various neoplasms, including those with poor prognosis. More research is needed on cell lines, model organisms, and possibly in the future also on humans.

Address for correspondence: Paweł Zapolnik, Students' Scientific Association of Clinical Genetics, Department of Clinical Genetics, Medical College, University of Rzeszów, Kopisto 2a, 35-959, Rzeszów, Poland

E-mail: pawel.zapolnik@onet.pl

Received: 29.08.2020; accepted: 30.09.2020; first published: 19.10 .2020

\section{OBJECTIVE}

MicroRNAs are a large group of small regulatory molecules that have essential functions in the cell. Understanding their functioning and influence on the neoplastic process may contribute to introducing new therapeutic methods in oncology. The aim of the review is to present the current knowledge of the miR-26a-5p molecule, its influence on various types of cancers, and to consider its use as an anticancer agent.

\section{DESCRIPTION OF THE STATE OF KNOWLEDGE}

MicroRNA-26a-5p is a molecule consisting of 22 nucleotides (Fig. 1). It is now known that it has many functions, of which one of the more interesting seems to be related to neoplasm, but discovering the role of miR-26a-5p in other biological processes can also bring many benefits to modern medicine.

3'-U-C-G-G-A-U-A-G-G-A-C-C-U-A-A-U-G-A-A-C-U-U- 5'

Figure 1. Nucleotide sequence of microRNA-26a-5p

The first cancer worth focusing on in the context of miR26a-5p is pancreatic cancer, one of the most aggressive and poor prognosis malignant neoplasms [7]. Wang et al. performed a study on tissue samples from 96 patients with pancreatic ductal adenocarcinoma (PDAC) and cell lines of 
this cancer. A possible oncogene, ARNTL2, was detected. It activates the TGF/BETA pathway and contributes to tumour progression. The authors showed that miR-26a-5p expression is reduced in PDAC, while the molecule itself binds to the 3'UTR region of ARNTL2 mRNA and causes its downregulation. Thanks to this process, tumour growth was inhibited and its invasiveness was reduced. Similar results were obtained in a study on mice with subcutaneous tumour xenografts [6].

The above research indicates the suppressor nature of miR-26a-5p and its possible use as an inhibitor of tumour proliferation. Zhang et al. conducted another study on the effects of miR-26a-5p on pancreatic cancer. In this case, the upregulated gene was SNHG6, the silencing of which led to inhibition of proliferation and activation of apoptosis in pancreatic cancer cells. This gene probably functions by regulating the FUBP1 protein. Increased expression of miR26a-5 downregulated FUBP1, which resulted in the inhibition of tumour proliferation [8]. This is another study supporting the evidence for a suppressive function of miR-26a-5p.

Another cancer associated with miR-26a-5p is papillary thyroid carcinoma. Shi et al. performed studies on primary papillary thyroid carcinoma tissues from 58 patients and a cell line. The expression of miR-26a-5p in cancer cells was decreased compared to normal thyroid tissue. The upregulation of miR-26a-5p inhibited tumour cell proliferation and depressed colony-forming ability, an effect achieved by inhibiting the expression of a direct target, in this case Wnt5a. These results were confirmed in an in vivo experiment in which miR-26a-5p inhibited tumour growth in a mouse model with subcutaneous xenograft [9].

Huang et al. assessed the effect of microRNA-26a-5p on breast cancer cells. Statistical analysis showed that in breast cancer, there is a decreased expression of miR-26a-5p, which is associated with a poorer prognosis. On the other hand, enhancing the expression of this molecule leads to the upregulation of $\mathrm{p} 21, \mathrm{p} 27$, and $\mathrm{p} 53$ proteins, control of the cell cycle, and inhibition of cell growth. miR-26a-5p binds to the 3'UTR region of the mRNA for the oncogenic RNF6 protein. This process inhibits its formation, which leads to a reduction in the expression of ERa and Bcl-xL [10]. Similar results were obtained by authors studying prostate cancer. Analogously, decreased expression of miR-26a-5p appears in prostate cancer cells, while its upregulation inhibited proliferation and metastasis. The direct target for miR-26a-5p was the SERBP1 protein, the silencing of which in prostate cancer was associated with metastasis and a higher rate in the Gleason scale [11].

The involement of miR-26a-5p in the pathogenesis of haematological neoplasms is also known. Jiang et al. performed studies on patient-derived acute myeloid leukemia (AML) cells and showed a decreased expression of miR-26a$5 \mathrm{p}$ and miR-23b-3p in leukemic cells. The common target for both of these molecules is peroxiredoxin III (PRXIII), a protein with antioxidant properties [12]. The elevated level of reactive oxygen species (ROS) likely acts as a trigger for haematopoietic stem cells to differentiate into the myeloid lineage [13]. It is possible that the downregulation of miR$26 \mathrm{a}-5 \mathrm{p}$ results in a reduction in the amount of ROS and impaired haematopoiesis, which may lead to the development of AML [12].

An interesting fact is also the connection of miR-26a-5p with cancer of embryonic origin - hepatoblastoma [14].
Zhang et al. discovered new targets for the miR-26a-5p molecule, the LIN28B protein, and serine-threonine Aurora kinase A (AURKA). Both proteins were characterized by increased expression in hepatoblastoma cells, and their silencing by miR-26a-5p resulted in the inhibition of cell proliferation and reduced colony formation in cell culture [15]. Ghanbari et al. analyzed the microRNA expression profile in the blood of 61 patients with recently diagnosed colorectal cancer. Compared to the control group of healthy people, the expression of miR-26a-5p was significantly lower in the cancer patients [16].

All the above studies indicate the suppressor nature of the microRNA-26a-5p; however, it is worth taking a closer look at the research proving the oncogenic role of this molecule in the carcinogenesis. Yu et al. assessed osteosarcoma cells which, contrary to the studies mentioned above, revealed an increased expression of miR-26a-5p in the cells of this tumour: miR-26a-5p downregulated the HOXA5 protein, which inhibits proliferation and cell cycle, and promotes paclitaxel-induced cell apoptosis [5]. MicroRNA-26a-5p is associated with numerous proteins involved in oncogenesis. A summary of connections of miR-26a-5p and various

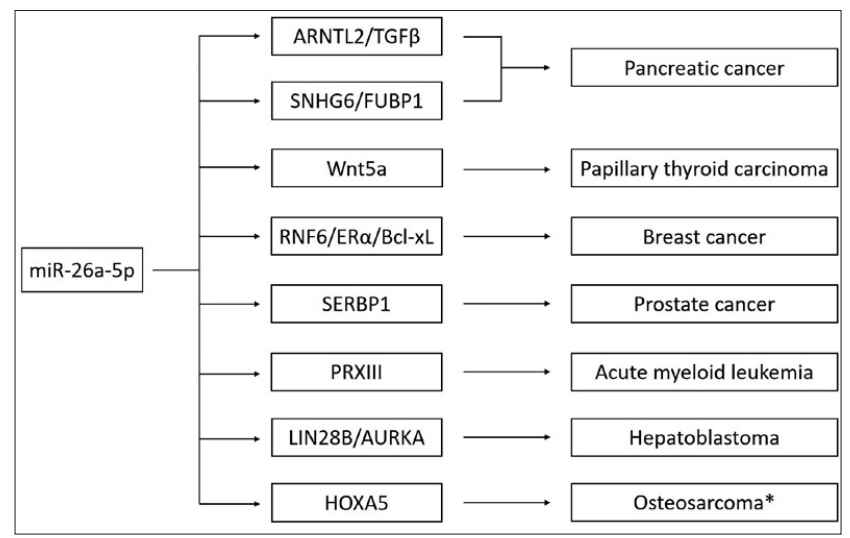

neoplasms is presented in Figure 2.

Figure 2. The linkage between microRNA-26a-5p and various direct targets and tumors.

*In most of the presented cases, miR-26a-5p acts as a tumor suppressor. In osteosarcoma cells, it shows an oncogenic nature

In addition to the fascinating topic of neoplasia, microRNA$26 \mathrm{a}-5 \mathrm{p}$ is also involved in other biological processes associated with non-cancerous human diseases.

The first disease worth attention in the context of miR-26a$5 \mathrm{p}$ is osteoarthritis. Rasheed et al. determined in their study that miR-26a-5p inhibits inducible nitric oxide synthase (iNOS), which takes part in the pathogenesis of this disease [17]. Jin et al. showed that miR-26a-5p derived from the exosomes of bone mesenchymal stem cells reduces the expression of the PTGS2 gene encoding cyclooxygenase 2 , and thus inhibits the development of osteoarthritis [18]. Zhang et al. conducted a study using the rat model of intracranial haemorrhage. Perihaematomal tissues showed decreased expression of miR-26a-5p and increased expression of the RANBP9 protein, which was found to be a direct target of the microRNA molecule. The upregulation of miR-26a-5p silenced the RANBP9, improved neurological functions, and inhibited neuronal apoptosis [19].

Another study on rats concerned myocardial inflammation after a percutaneous coronary intervention (PCI). Kong 
et al. revealed that the expression of miR-26a-5p decreased after microembolization. In contrast, its increase led to improved function and reduction of inflammation in the myocardium by silencing the target protein HMGA1 [20]. Duan et al. analyzed a mouse model of diabetic nephropathy. They demonstrated the decreased expression of miR-26a$5 \mathrm{p}$ and increased Toll-like receptor type 4 (TLR4). miR$26 a-5 p$ derived from extracellular vesicles from adipose mesenchymal stem cells decreased TLR4 expression, which inhibited the NF- $\kappa \mathrm{B}$ pathway and downregulated VEGF-A. This mechanism protected the mouse cells from high glucoseinduced damage [21].

The above works prove the reduced expression of miR$26 a-5 p$ in various pathological states and the beneficial effects of enhancing this molecule. There are also reports of a negative role of miR-26a-5p in disease development. Müller-Deile et al., in a study on human podocytes culture, found that miR-26a-5p, through the PIK3C $2 a$ protein, reduces the expression of VEGF-A, which leads to podocyte damage and the development of preeclampsia. Analysis of zebrafish (Danio rerio) showed that miR-26a-5p, by inhibiting VEGF-A, causes generalized oedema and proteinuria. The authors additionally conducted a small pilot study on a group of 23 patients with preeclampsia and showed increased levels of miR-26a-5p in the urine compared to a control group [22].

Huang et al. conducted a study on synovial tissue obtained from patients with rheumatoid arthritis (RA), osteoarthritis, and after joint trauma. The expression of miR-26a-5p in fibroblast-like synoviocytes in RA was increased compared to other synovial pathologies. miR-26a-5p in RA fibroblastlike synoviocytes promoted the transition from the G1 to the $S$ phase of the cell cycle. Increased expression of miR26a-5p inhibited PTEN protein and stimulated the PI3K/ AKT pathway, which resulted in increased proliferation and invasion of fibroblast-like synoviocytes, which play an essential role in the pathogenesis of RA [23].

Another topic of interest is the role of miR-26a-5p in liver regeneration. Transplantation of mesenchymal stem cells (MSCs) in cases of liver damage can lead to better recovery. MSCs produce a hepatocyte growth factor (HGF) which protects against cirrhosis. Chen et al. assessed the role of miR-26a-5p in this context in a mouse model. The authors showed that the amount of HGF protein in MSCs is low, while HGF mRNA expression is high. It is associated with an increase in miR-26a-5p expression and inhibition of the direct target of HGF. The suppression of the miRNA molecule reduces liver fibrosis and portal hypertension [24]. Figure 3 shows the participation of miR-26a-5p in nonneoplastic diseases.

\section{CONCLUSIONS}

Knowledge of microRNAs is continuously growing. Numerous studies have indicated the critical role of microRNA-26a-5p in the development of human diseases, both cancerous and noncancerous. Thanks to this, the prospect of using miR-26a$5 \mathrm{p}$ as a prognostic factor becomes a reality. Multiple studies indicate that this molecule can be used as a therapeutic agent to inhibit the development of diseases by influencing various signaling pathways. It will probably also be possible to use miR-26a-5p inhibitors in those entities in which increased expression is disadvantageous. It is necessary to conduct

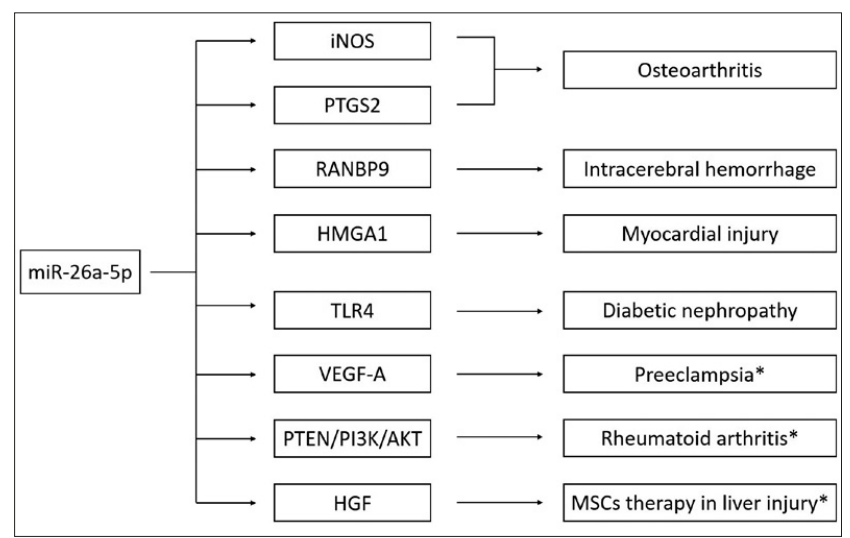

Figure 3. The linkage between microRNA-26a-5p and various noncancerous diseases

* In most of the presented cases, increased expression of miR-26a-5p is beneficial and can be used therapeutically. In preeclampsia, RA, and the use of MSCs, increased expression of miR-26a-5p has a detrimental effect

further studies to assess the functions and therapeutic applicability of miR-26a-5p in laboratory models. Due to this, safe clinical trials in humans can be performed in the future.

However, the current knowledge about miR-26a-5p has some limitations. Most of the research is carried out in cell lines and model organisms, which may represent some kind of the molecule's activity, but do not exactly correspond to its in vivo behaviour in humans. The vast majority of the research on miR-26a-5p comes from China. Performing similar trials on other samples could throw new light on this topic because the microRNA expression profile can vary between populations. An additional limitation may be the fact of using miR-26a-5p molecules, difficulties in delivering them to a specific tissue, and ensuring proper activity for a direct target. Despite the limitations, it seems appropriate to conduct further studies on miR-26a-5p, which may allow the accurate assessment of the potential therapeutic effect and safety of this molecule.

\section{Acknowledgment}

The authors thank Karolina Szela for her help in collecting materials.

\section{REFERENCES}

1.Xie T, Huang M, Wang Y, et al. MicroRNAs as Regulators, Biomarkers and Therapeutic Targets in the Drug Resistance of Colorectal Cancer. Cell Physiol Biochem. 2016; 40(1-2): 62-76. https://doi. org/10.1159/000452525

2. Li Z, Lin C, Zhao L, et al. Oncogene miR-187-5p is associated with cellular proliferation, migration, invasion, apoptosis and an increased risk of recurrence in bladder cancer. Biomed Pharmacother. 2018; 105 : 461-469. https://doi.org/10.1016/j.biopha.2018.05.122

3. Bartel DP. MicroRNAs: target recognition and regulatory functions. Cell. 2009; 136(2): 215-233. https://doi.org/10.1016/j.cell.2009.01.002

4. Wu L, Belasco JG. Let me count the ways: mechanisms of gene regulation by miRNAs and siRNAs. Mol Cell. 2008; 29(1): 1-7. https:// doi.org/10.1016/j.molcel.2007.12.010

5. Yu T, Chen D, Zhang L, et al. microRNA-26a-5p Promotes Proliferation and Migration of Osteosarcoma Cells by Targeting HOXA5 in vitro and in vivo. Onco Targets Ther. 2019; 12: 11555-11565. https://doi. org/10.2147/OTT.S232100

6. Wang Z, Liu T, Xue W, et al. ARNTL2 promotes pancreatic ductal adenocarcinoma progression through TGF/BETA pathway and is regulated by miR-26a-5p. Cell Death Dis. 2020; 11(8): 692. https://doi. org/10.1038/s41419-020-02839-6 
7. Yang Y, Sun Y, Wang H, et al. MicroRNA-221 induces autophagy through suppressing HDAC6 expression and promoting apoptosis in pancreatic cancer. Oncol Lett. 2018; 16(6): 7295-7301. https://doi. org/10.3892/ol.2018.9513

8. Zhang XX, Chen H, Li HY, et al. Long non-coding RNA small nucleolar RNA host gene 6 aggravates pancreatic cancer through upregulation of far upstream element binding protein 1 by sponging microRNA-26a5p. Chin Med J (Engl). 2020; 133(10): 1211-1220. https://doi.org/10.1097/ CM9.0000000000000758

9. Shi D, Wang H, Ding M, et al. MicroRNA-26a-5p inhibits proliferation, invasion and metastasis by repressing the expression of Wnt5a in papillary thyroid carcinoma. Onco Targets Ther. 2019; 12: 6605-6616. https://doi.org/10.2147/OTT.S205994

10. Huang ZM, Ge HF, Yang CC, et al. MicroRNA-26a-5p inhibits breast cancer cell growth by suppressing RNF6 expression. Kaohsiung J Med Sci. 2019; 35(8): 467-473. https://doi.org/10.1002/kjm2.12085

11. Guo K, Zheng S, Xu Y, et al. Loss of miR-26a-5p promotes proliferation, migration, and invasion in prostate cancer through negatively regulating SERBP1. Tumour Biol. 2016; 37(9): 12843-12854. https://doi.org/10.1007/ s13277-016-5158-z

12. Jiang W, Min J, Sui X, et al. MicroRNA-26a-5p and microRNA-23b$3 p$ up-regulate peroxiredoxin III in acute myeloid leukemia. Leuk Lymphoma. 2015; 56(2): 460-471. https://doi.org/10.3109/10428194. 2014.924115

13. Tothova Z, Gilliland DG. FoxO transcription factors and stem cell homeostasis: insights from the hematopoietic system. Cell Stem Cell 2007; 1(2): 140-152. https://doi.org/10.1016/j.stem.2007.07.017

14.Zhang YT, Feng LH, Zhong XD, et al. Vincristine and irinotecan in children with relapsed hepatoblastoma: a single-institution experience. Pediatr Hematol Oncol. 2015; 32(1): 18-25. https://doi.org /10.3109/08880018.2014.909913

15. Zhang Y, Zhao Y, Wu J, et al. MicroRNA-26-5p functions as a new inhibitor of hepatoblastoma by repressing lin-28 homolog B and aurora kinase a expression. Hepatol Commun. 2018; 2(7): 861-871. https://doi. org/10.1002/hep4.1185
16. Ghanbari R, Mosakhani N, Asadi J, et al. Downregulation of Plasma MiR-142-3p and MiR-26a-5p in Patients With Colorectal Carcinoma. Iran J Cancer Prev. 2015; 8(3): e2329. https://doi.org/10.17795/ijcp2329 17. Rasheed Z, Al-Shobaili HA, Rasheed N, et al. MicroRNA-26a-5p regulates the expression of inducible nitric oxide synthase via activation of NF- $\kappa \mathrm{B}$ pathway in human osteoarthritis chondrocytes. Arch Biochem Biophys. 2016; 594: 61-67. https://doi.org/10.1016/j.abb.2016.02.003

18. Jin Z, Ren J, Qi S. Human bone mesenchymal stem cells-derived exosomes overexpressing microRNA-26a-5p alleviate osteoarthritis via down-regulation of PTGS2. Int Immunopharmacol. 2020; 78: 105946. https://doi.org/10.1016/j.intimp.2019.105946

19.Zhang H, Lu X, Hao Y, et al. MicroRNA-26a-5p alleviates neuronal apoptosis and brain injury in intracerebral hemorrhage by targeting RAN binding protein 9. Acta Histochem. 2020; 122(5): 151571. https:// doi.org/10.1016/j.acthis.2020.151571

20. Kong B, Qin Z, Ye Z, et al. microRNA-26a-5p affects myocardial injury induced by coronary microembolization by modulating HMGA1. J Cell Biochem. 2019; 120(6): 10756-10766. https://doi.org/10.1002/jcb.28367 21. Duan Y, Luo Q, Wang Y, et al. Adipose mesenchymal stem cell-derived extracellular vesicles containing microRNA-26a-5p target TLR4 and protect against diabetic nephropathy [published online ahead of print, 2020 Jun 24]. J Biol Chem. 2020; jbc.RA120.012522. https://doi. org/10.1074/jbc.RA120.012522

22. Müller-Deile J, Schröder P, Beverly-Staggs L, et al. Overexpression of preeclampsia induced microRNA-26a-5p leads to proteinuria in zebrafish. Sci Rep. 2018; 8(1): 3621. https://doi.org/10.1038/s41598018-22070-w

23. Huang Z, Xing S, Liu M, et al. MiR-26a-5p enhances cells proliferation, invasion, and apoptosis resistance of fibroblast-like synoviocytes in rheumatoid arthritis by regulating PTEN/PI3K/AKT pathway. Biosci Rep. 2019; 39(7): BSR20182192. https://doi.org/10.1042/BSR20182192

24. Chen L, Zeng W, Yang B, et al. Expression of antisense of microRNA26a-5p in mesenchymal stem cells increases their therapeutic effects against cirrhosis. Am J Transl Res. 2017; 9(3): 1500-1508. 